\title{
Size of third and fourth ventricle in obstructive and communicating acute hydrocephalus after aneurysmal subarachnoid hemorrhage
}

\author{
D. S. Knol • J. van Gijn • \\ C. L. J. J. Kruitwagen · G. J. E. Rinkel
}

Received: 10 March 2010/Revised: 24 June 2010/ Accepted: 14 July 2010/Published online: 3 August 2010

(C) The Author(s) 2010. This article is published with open access at Springerlink.com

\begin{abstract}
In patients with acute hydrocephalus after aneurysmal subarachnoid haemorrhage (SAH), lumbar drainage is possible if the obstruction is in the subarachnoid space (communicating hydrocephalus). In case of intraventricular obstruction (obstructive hydrocephalus), ventricular drainage is the only option. A small fourth ventricle is often considered a sign of obstructive hydrocephalus. We investigated whether the absolute or relative size of the fourth ventricle can indeed distinguish between these two types of hydrocephalus. On CT-scans of 76 consecutive patients with acute headache but normal CT and CSF, we measured the cross-sectional surface of the third and fourth ventricle to obtain normal planimetric values. Subsequently we performed the same measurements on 117 consecutive SAH patients with acute hydrocephalus. These patients were divided according to the distribution of blood on CT-scan into three groups: mainly intraventricular blood $(n=15)$, mainly subarachnoid blood $(n=54)$ and both intraventricular and subarachnoid blood $(n=48)$. The size of the fourth ventricle exceeded the upper limit of normal in 2 of the $6(33 \%)$ patients with intraventricular blood but without haematocephalus, and in 15 of the $54(28 \%)$ patients with mainly subarachnoid blood. The mean ratio between the third and fourth ventricle was 1.45 (SD 0.66) in patients with intraventricular blood and 1.42 (SD 0.91) in those with mainly
\end{abstract}

D. S. Knol · J. van Gijn $(\bowtie) \cdot$ G. J. E. Rinkel

Department of Neurology, Rudolf Magnus Institute of Neuroscience, University Medical Centre Utrecht, PO box 85500, 3508 GA Utrecht, The Netherlands e-mail: j.vangijn@umcutrecht.nl

C. L. J. J. Kruitwagen

Julius Centre for Health Sciences and Primary Care,

University Medical Centre Utrecht, Utrecht, The Netherlands subarachnoid blood. Neither fourth ventricular size nor the ratio between the third and fourth ventricles discriminates between the two groups. A small fourth ventricle does not necessarily accompany obstructive hydrocephalus and is therefore not a contraindication for lumbar drainage.

Keywords Subarachnoid haemorrhage - Cerebral aneurysm · Acute hydrocephalus

\section{Introduction}

Acute hydrocephalus complicates subarachnoid haemorrhage (SAH) from a ruptured aneurysm in about $20 \%$ of the patients [1]. Acute hydrocephalus can be treated by means of lumbar puncture or drainage when the site of obstruction is in the subarachnoid space (communicating hydrocephalus), but when the obstruction is intraventricular (obstructive hydrocephalus) external ventricular drainage (EVD) is the only option. EVD should preferably not be applied in case of communicating acute hydrocephalus because it carries a substantial risk of complications such as misplacement, ventriculostomy-related hemorrhage, and ventriculostomy-related infections [2]. It is therefore important to discriminate between these two types of hydrocephalus, but unfortunately this distinction is often not straightforward. Only in patients with massive extension of the haemorrhage in the third or fourth ventricle or in patients with no intraventricular blood at all the distinction seems reasonably clear.

Theoretically, it might be supposed that the third and fourth ventricles increase in size when the site of obstruction is in the subarachnoid space, but not when the obstruction of the CSF circulation is upstream in the ventricular system. A similar assumption is that relatively 
greater enlargement of the third ventricle than of the fourth ventricle is characteristic of obstruction at the level of the cerebral aqueduct. To date there are no data to support or refute these assumptions. In patients already presenting with acute hydrocephalus as a result of massive intraventricular hemorrhage external ventricular drainage seems indeed the only option, regardless of the size of the third or fourth ventricle. In patients with partially filled ventricles it can be more difficult to estimate the site of blockage and therefore also the optimal treatment. Ventricular size might be helpful in such cases in indicating the most effective treatment.

However, little is known about normal values for the size of the third and fourth ventricles; most studies performed with Computed Tomography (CT) scanning used linear indices [3-6]. Some studies addressed the prognostic significance of intraventricular haemorrhage, but did not distinguish the type of hydrocephalus according to the site of obstruction [7, 8].

Our aim was to measure the planimetric size of the third and fourth ventricles, firstly in normal subjects and subsequently in two defined subsets of patients with acute hydrocephalus after aneurysmal SAH: those with mainly subarachnoid blood (presumed non-obstructive) and those with mainly intraventricular blood (presumed obstructive). If clear differences can be found in the size of the third and fourth ventricle between these two subsets of patients, this difference may help future decisions about the optimal mode of treatment in patients in whom the distribution of blood provides equivocal information about the site of obstruction of the CSF flow.

\section{Patients and methods}

To measure the normal sizes for third and fourth ventricle, we used CT scans of 76 consecutive patients who had been admitted between 2003 and 2007 to the accident and emergency department of the University Medical Centre Utrecht because of sudden, severe headache, but in whom $\mathrm{SAH}$ or other structural disorders had been subsequently ruled out by CT scanning and a diagnostic lumbar puncture. This period was chosen because digital archiving of radiological studies started in 2003, which was necessary for our computerised measurements. Subsequently we retrieved from our prospectively collected database of patients with SAH from the same period those patients with an aneurysmal cause of the SAH $(n=600)$ who had enlargement of the lateral ventricles on any CT scan (bicaudate index on any CT slice exceeding the 95th percentile for age) [1]. Of the 600 patients 140 (23\%) fulfilled this criterion. We excluded patients in whom the first scan had been made more than 3 days after onset of the symptoms, because blood can be rapidly cleared from the subarachnoid space [9]. We also excluded patients in whom a ventricular catheter had already been placed before admission to our centre.

According to our institutional protocol, the orientation of the CT scan slices was always with an inclination of $20^{\circ}$ above the orbitomeatal plane, with a slice thickness of 5 or $6 \mathrm{~mm}$. We measured the cross-sectional surface of the third and fourth ventricle on the slice with the largest area, with a standard computer program (Mirador ${ }^{\circledR}$ ) that automatically corrects for magnification. Subsequently we studied the quantity and distribution of extravasated blood on the admission CT scan, to discriminate between obstructive or communicating hydrocephalus. Therefore, we graded the amount of blood in each ventricle, and separately for each of the perimesencephalic cisterns, Sylvian fissures and the suprasellar cisterns [10]. Patients were then divided into three groups (Fig. 1): firstly patients with mostly intraventricular haemorrhage, secondly patients in whom the haemorrhage was (almost) completely confined to the subarachnoid space, and lastly a combined group, with large amounts of blood in the ventricles as well as in the basal cisterns.

We assumed that hydrocephalus in patients with predominantly intraventricular blood was of the obstructive type, and in those with mainly subarachnoid blood of the non-obstructive (communicating) type. In the group with intraventricular haemorrhage we accepted moderate amounts of blood in the interpeduncular fossa, suprasellar cisterns or in a single Sylvian fissure (or small amounts in both). To the combined group, in whom the site of obstruction was ambiguous, we also assigned patients with predominant extravasation in the subarachnoid space but with moderate amounts of blood in or near the foramina of Monro (including haematomas in the septum pellucidum) and at the posterior end of the third ventricle, near the aqueduct, since these clots might interfere with CSF flow, even if they might be explained by sedimentation. We assumed no such obstruction if blood was detected in the suprapineal recess.

\section{Data analysis}

To correct for right-sided skewness, we performed a log transformation on our data. Next we estimated $95 \%$ confidence intervals with a $90 \%$ outer interval for mean ventricular size in our control group of patients with acute headache but no SAH and in the groups with hydrocephalus after $\mathrm{SAH}$. We also estimated the mean ratio between the size of the third and fourth ventricle in the control group, in patients with mainly intraventricular blood and in patients with mainly subarachnoid blood. We separately 
Fig. 1 Example of each of the three groups in which patients with acute hydrocephalus after aneurysmal haemorrhage were categorised

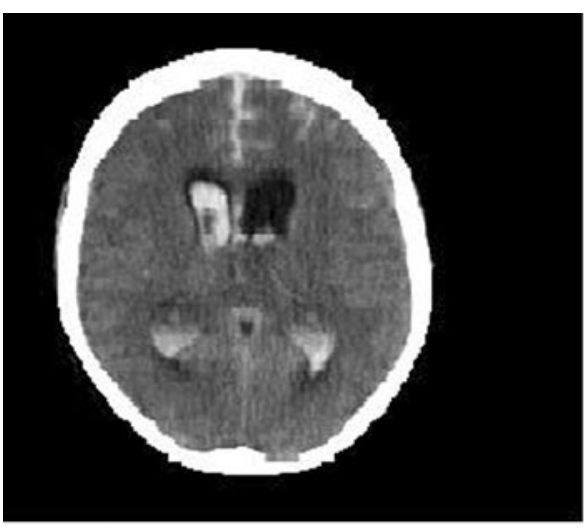

mainly intraventricular blood

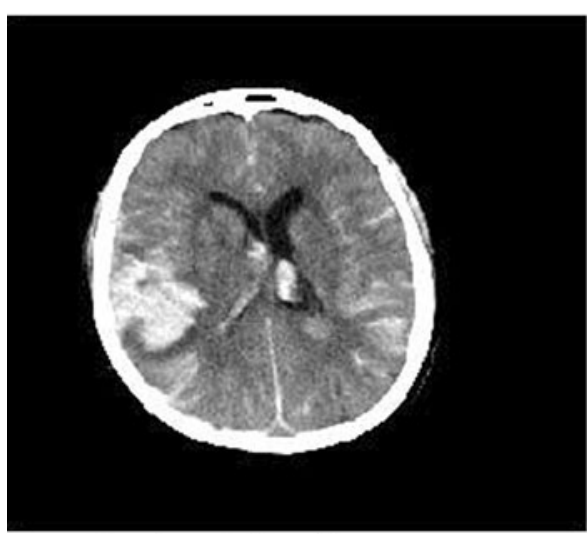

combined type

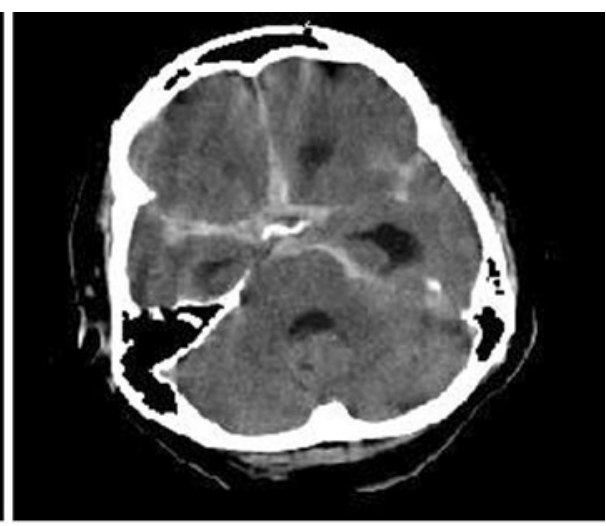

mainly subarachnoid blood identified patients with mainly intraventricular blood in whom intraventricular haemorrhage also involved the third and fourth ventricle (haematocephalus), because in these patients any ventricular expansion might have been caused directly by the force of the initial haemorrhage, instead of indirectly, by obstruction of CSF flow.

\section{Results}

\section{Ventricular size in control group}

Of the 76 patients with sudden headache but normal CT and CSF examinations, 49 (64\%) were women. Their mean age was 43.9 years (SD 14.6).

The mean size (maximum cross-sectional surface) of the third ventricle was $58 \mathrm{~mm}^{2}$ (95\% CI 10-218) and that of the fourth ventricle $119 \mathrm{~mm}^{2}$ (95\% CI 52-242). Distinction between different age groups had no effect on the right tail of the distribution, for which reason we analysed all subjects together. Figure 2 shows the distribution of the measurements.

The mean ratio between the sizes of the third and fourth ventricle was 0.5 (95\% CI 0.1-1.9).
Size of the third and fourth ventricles in hydrocephalus after $\mathrm{SAH}$

Of the 140 patients with enlarged ventricles after SAH, 117 fulfilled the inclusion criteria. Their mean age was 61.0 years; $70 \%$ were women. Of these 117 patients, we classified 15 patients as mainly intraventricular blood, 54 as mainly subarachnoid blood and 48 as combined. Table 1 shows the demographic characteristics for the three groups, according to the site of obstruction.

The mean ventricular size for all three groups is shown in Table 1. In some patients dilatation of the third or fourth ventricle might be explained, entirely or partly, by expansion through direct haemorrhage into the ventricular system. When we excluded these patients with haematocephalus, the sizes of the third or fourth ventricle were similar in patients with mainly intraventricular and those with mainly subarachnoid blood. The size of the fourth ventricle exceeded the upper limit of the $95 \%$ confidence interval for controls in 2 of the $6(33 \%)$ patients in the group with mainly intraventricular blood but without haematocephalus and in 15 of the 54 patients (28\%) group with mainly subarachnoid blood (Fig. 3). For the third ventricle, the size exceeded the upper limit of the $95 \%$ 

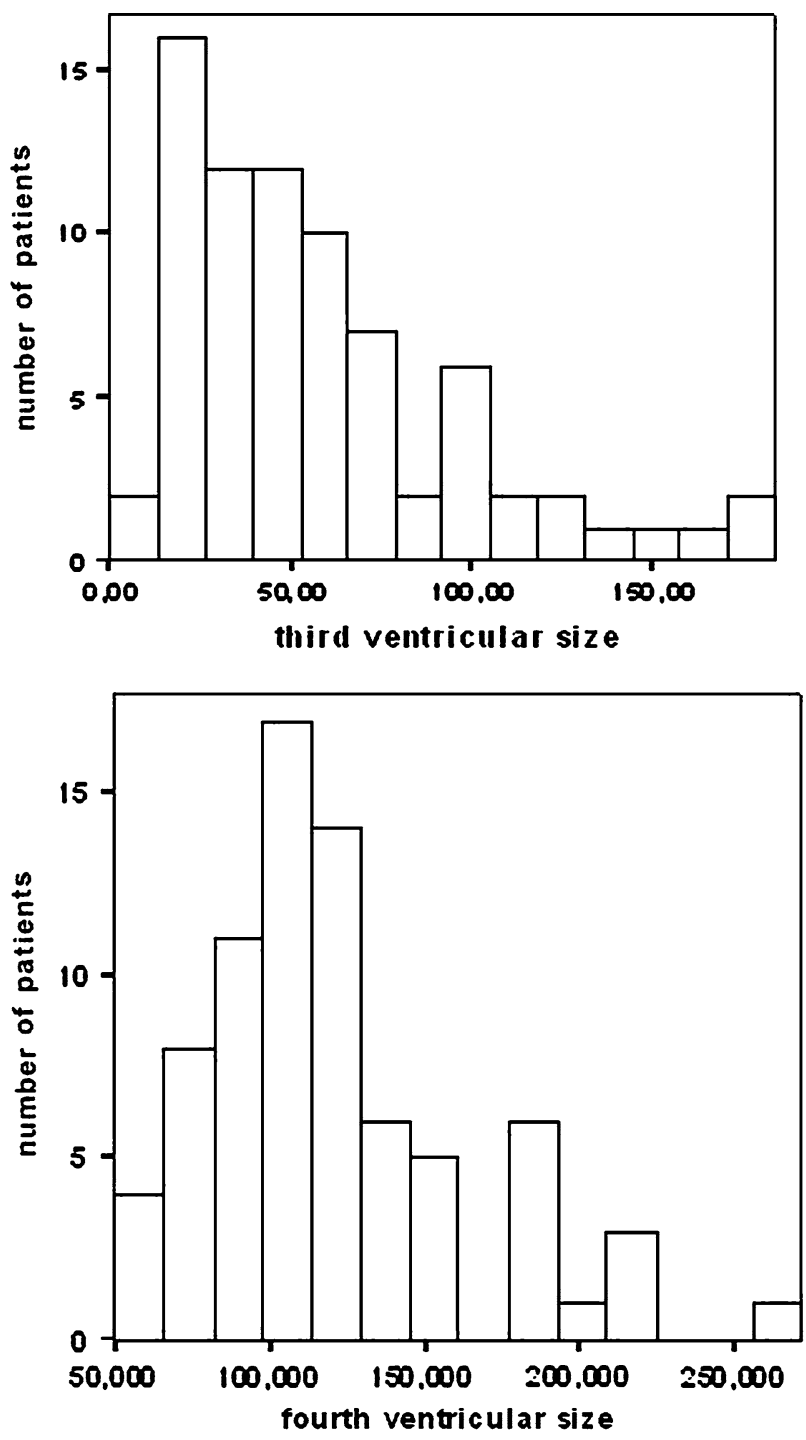

Fig. 2 Graphs showing the distribution of the size (maximal crosssectional area in the axial plane in $\mathrm{mm}^{2}$ ) of the third ventricle (upper panel) and fourth ventricle (lower panel) in 76 controls, as measured on computed tomography scanning

confidence limit for normal subjects in 3 of the 4 patients $(75 \%)$ in the group with mainly intraventricular blood without haematocephalus, and in 32 of the 54 patients $(59 \%)$ in the group with mainly subarachnoid blood.
Ratio between the size of the third and fourth ventricle

After exclusion of patients with haematocephalus, the mean ratio between the largest surface area of the third ventricle and that of the fourth ventricle was 1.45 (SD 0.66) in the group with mainly intraventricular blood and 1.42 (SD 0.91) in the group with mainly subarachnoid blood. Compared with controls, 10 (19\%) of the 54 patients in the group with mainly subarachnoid blood had a ratio above the $95 \%$ CI for controls, against 2 of the 15 patients (13\%) in the group with mainly intraventricular blood.

\section{Discussion}

We found that in patients with hydrocephalus after SAH the size of neither the fourth nor the third ventricle differs between patients with mainly intraventricular or those with mainly subarachnoid blood. Because the distinction between an obstructive or communicating hydrocephalus after SAH usually is made upon the distribution of blood, this implies that the size of neither the fourth nor the third ventricle is useful in making this distinction. Also the ratio between the size of the third and fourth ventricle is not characteristic of either type of hydrocephalus.

The third ventricle showed a greater increase in size (compared with values for controls) than the fourth ventricle, both in patients with mainly subarachnoid and patients with mainly intraventricular blood. A greater enlargement of the third than of the fourth ventricle is therefore compatible with a CSF block in the subarachnoid space and should not exclude lumbar drainage as treatment option for hydrocephalus.

A dilated fourth ventricle was found in 15 of 54 patients with mainly subarachnoid blood patients and in 2 of the 15 patients with mainly intraventricular blood, but the predictive value of this finding cannot be estimated because almost half of all 117 patients with acute dilatation of the lateral ventricles had to be assigned to the combined group, in which the site of CSF obstruction could not be deduced from the distribution of blood on the CT scan. The same conclusion applies to the third ventricle and the ratio for the size of the two ventricles.

Table 1 Characteristics and mean ventricular size of third and fourth ventricle according to the presumed site of obstruction in the CSF flow

\begin{tabular}{|c|c|c|c|c|}
\hline & $\begin{array}{l}\text { Only intraventricular } \\
\text { blood, all patients } \\
n=15\end{array}$ & $\begin{array}{l}\text { Only intraventricular blood, } \\
\text { without haematocephalus } \\
n=6\end{array}$ & $\begin{array}{l}\text { Only subarachnoid } \\
\text { blood } n=54\end{array}$ & $\begin{array}{l}\text { Undetermined } \\
n=48\end{array}$ \\
\hline Mean age & $52.2(9.4)$ & $48.8(7.6)$ & $60.3(13.9)$ & $64.4(12.8)$ \\
\hline Women $(\%)$ & $10(67 \%)$ & $3(75 \%)$ & $35(65 \%)$ & $37(77 \%)$ \\
\hline Third ventricular size median (IQR) & $358.0(195.3)$ & $314.8(191.5)$ & $233.4(125.5)$ & $276.1(149.8)$ \\
\hline Fourth ventricular size median (IQR) & $350.3(257.8)$ & $217.6(64.2)$ & $174.3(107.4)$ & $195.5(120.2)$ \\
\hline Ratio 3rd/4th ventricle mean (SD) & $1.05(0.45)$ & $1.45(0.66)$ & $1.42(0.91)$ & $1.61(1.32)$ \\
\hline
\end{tabular}



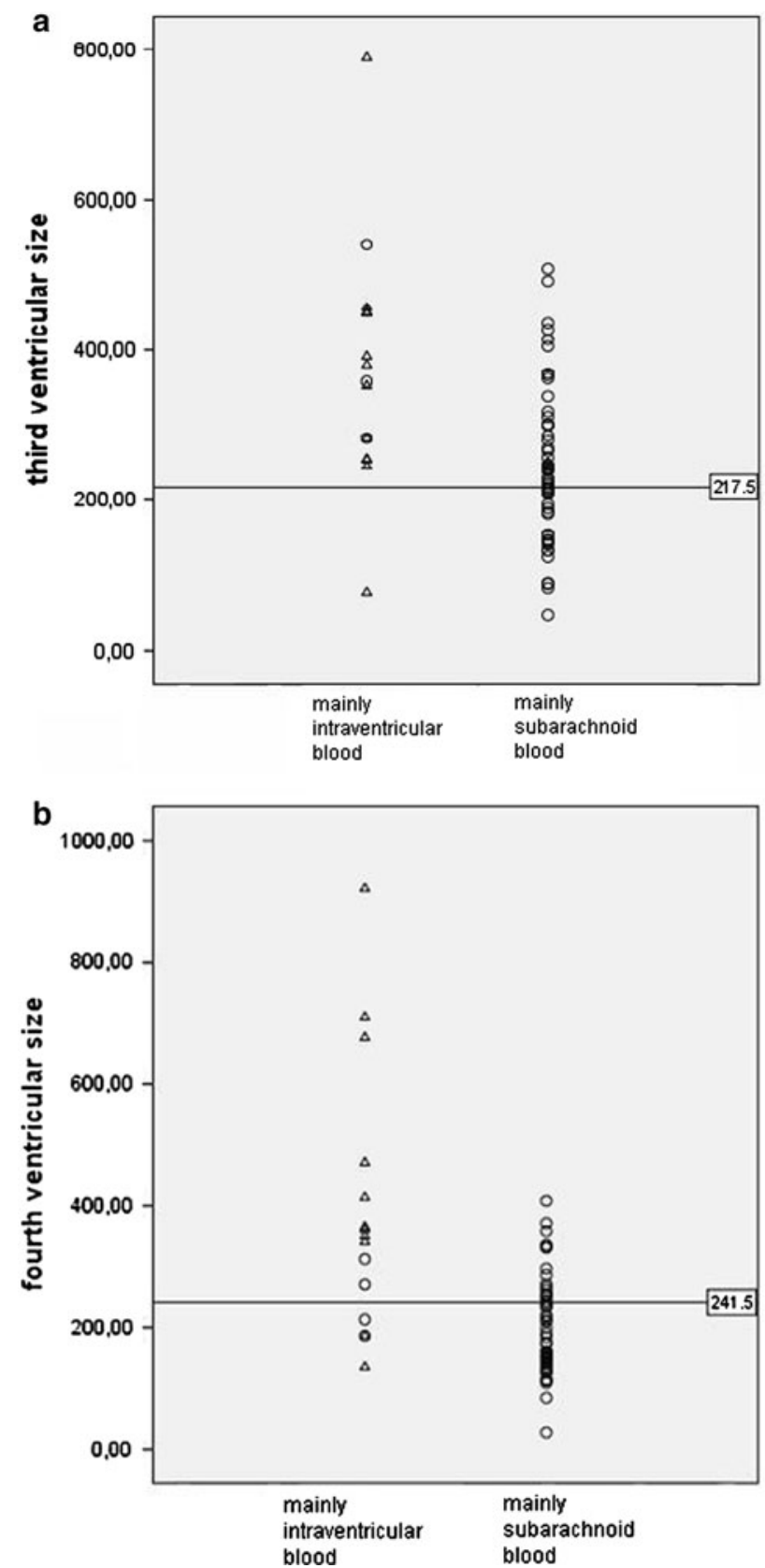

Fig. 3 Graphs showing the distribution of sizes of the third ventricle (a) and fourth ventricle (b), in $\mathrm{mm}^{2}$ (maximal cross-sectional surface), according to the site of obstruction (within the ventricular system or in the subarachnoid space), in 69 patients with acute dilatation of the lateral ventricles after SAH. Value for ventricles in which the cavity was completely filled (and probably expanded) by blood are represented by triangles instead of circles. The horizontal line represents the upper limit of normal

We chose planimetric measurements because it was convenient in practice, whereas previous studies of enlargement of the lateral ventricles used linear or volumetric measurements [3-7, 11]. Linear methods such as the bicaudate index are probably suitable for the lateral ventricles, but much less so for the fourth ventricle, because of its shape. Since measurements of a single slice can lead to large differences in results [12], we measured the areas of the third and fourth ventricles on all slices and only used the highest values. Volumetric measurements are of course more accurate than planimetry but less feasible, because of the much more complicated procedure. In theory the results might have been different if volumetric instead of planimetric measures had been used, because they are more accurate. We doubt whether they would show anything other than the same overlap in the sizes of the fourth and third ventricle between patients with obstruction within or outside the ventricular system after $\mathrm{SAH}$.

It often is difficult to distinguish between obstructive and communicating hydrocephalus on the basis of the distribution of blood on admission CT scans, since many patients with ruptured intracranial aneurysms show extensive extravasation in all CSF spaces as well as in the ventricular system. To ensure strict separation between obstructive and communicating hydrocephalus group we selected only those patients in whom the distribution of blood on CT-scanning clearly indicated obstruction in the subarachnoid space or in the ventricles. Because our criteria were necessarily strict, we were left with a large combined group of patients in whom we were uncertain about the site of the site of blockage. New techniques such as magnetic resonance phase contrast imaging may be helpful in determining the precise site of CSF flow obstruction and in estimating the type of hydrocephalus more accurately [13].

We conclude that the size of the fourth ventricle and the ratio between the third and fourth ventricle are not helpful in distinguishing between obstructive and communicating hydrocephalus if the site of obstruction is not evident from the patterns of haemorrhage on CT. A small fourth ventricle does not necessarily imply obstructive hydrocephalus and is not in itself a reason not to perform lumbar puncture or to insert a lumbar drain to treat symptomatic acute hydrocephalus.

Conflict of interest The authors declare that they have no conflict of interest.

Open Access This article is distributed under the terms of the Creative Commons Attribution Noncommercial License which permits any noncommercial use, distribution, and reproduction in any medium, provided the original author(s) and source are credited.

\section{References}

1. van Gijn J, Hijdra A, Wijdicks EFM et al (1985) Acute hydrocephalus after aneurysmal subarachnoid hemorrhage. J Neurosurg 63:355-362 
2. Saladino A, White JB, Wijdicks EFM et al (2009) Malplacement of ventricular catheters by neurosurgeons: a single institution experience. Neurocrit Care 10:248-252

3. Hamano K, Iwasaki N, Takeya T et al (1993) A comparative study of linear measurement of the brain and three-dimensional measurement of brain volume using CT scans. Pediatr Radiol 23:165-168

4. Mataro M, Poca MA, Sahuquillo J et al (2000) Cognitive change after cerebrospinal fluid shunting in young adults with spina bifida and assumed arrested hydrocephalus. J Neurol Neurosurg Psychiatry 68:615-621

5. O'Hayon BB, Drake JM, Ossip MG et al (1998) Frontal and occipital horn ratio: a linear estimate of ventricular size for multiple imaging modalities in pediatric hydrocephalus. Pediatr Neurosurg 29:245-249

6. Eide PK (2003) The relationship between intracranial pressure and size of cerebral ventricles assessed by computed tomography. Acta Neurochir 145:171-179

7. Shapiro SA, Campbell RL, Scully L (1994) Hemorrhagic dilatation of the fourth ventricle: an ominous predictor. J Neurol $80: 805-809$
8. Young WB, Lee KP, Pessin MS et al (1990) Prognostic significance of ventricular blood in supratentorial hemorrhage: a volumetric study. Neurology 40:616-619

9. van Gijn J, Kerr RS, Rinkel GJE (2007) Subarachnoid haemorrhage. Lancet 369:306-318

10. Hijdra A, Brouwers PJAM, Vermeulen M et al (1990) Grading the amount of blood on computed tomograms after subarachnoidal hemorrhage. Stroke 21:1156-1161

11. Sze RW, Ghioni V, Weinberger E et al (2003) Rapid computed tomography technique to measure ventricular volumes in the child with suspected ventriculoperitoneal shunt failure II. J Comput Asssist Tomogr 27:668-673

12. Woods BT, Douglass A, Gescuk B (1991) Is the VBR still a useful measure of changes in the cerebral ventricles? Psychiatry Res 40:1-10

13. Saliou G, Balédent O, Lehmann P et al (2009) Caractérisation des perturbations du flux de LCS dans l'aqueduc du mésencéphale par IRM-CP a la phase aigüe de l'hémorragie meningée. J Neuroradiol $36: 41-47$ 